Jean-Michel Jeannin

Beat Meier

\section{Internationale Tagung Phytotherapie 2014 in Winterthur: Phytotherapie in der Geriatrie und Gerontologie - ein Rückblick}

Das hochwertige wissenschaftliche Programm, die Posterpräsentation und die Rahmenveranstaltungen boten den Teilnehmenden viele Möglichkeiten, sich zu informieren und sich auszutauschen. Die technische Organisation lag in den erfahrenen Händen der Zürcher Hochschule für angewandte Wissenschaften (ZHAW), deren Mitarbeitende auch diese Herausforderung annähernd perfekt meisterten. Die für das wissenschaftliche Programm federführende Schweizerische Medizinische Gesellschaft für Phytotherapie (SMGP) versuchte, ihre Konzepte in Abstimmung mit den übrigen Verbänden darzustellen, weshalb der Hauptfokus auf die Therapie gelegt wurde. Dies insbesondere am ersten Tag der Veranstaltung, der sich dem Thema «Phytotherapie in der Geriatrie und Gerontologie» widmete.

\section{«Pflanzliche Antidepressiva und Anxiolytica beim älteren Patienten»}

Prof. Dr. med. Siegfried Kasper (Abb. 2), Medizinische Universität Wien, beschrieb die adäquate medizinische Versorgung eines immer älter werdenden polymorbiden Patientenkollektivs als eine Herausforderung für die Zukunft. Auf die Polymorbidität wird mit Polypharmakotherapie geantwortet, was insbesondere im ambulanten Bereich Risiken birgt. Bei Phytopharmaka sind, im Unterschied zu Synthetika, vom Wirkungsmechanismus bedingte Nebenwirkungen sehr selten oder sogar unbekannt. Für alle Arzneimittel gilt, dass nur wenige
Fünf nationale und internationale Fachgesellschaften für Phytotherapie und Arzneipflanzenforschung sowie drei renommierte Institute (Abb. 1) standen hinter dem gemeinsamen Kongress vom 18. bis 21. Juni 2014 in Winterthur. Mit annähernd 500 Teilnehmenden und Beteiligten - vorwiegend aus den drei deutschsprachigen Ländern, aber auch aus anderen Nationen - war dies der bisher grösste Phytotherapie-Kongress.

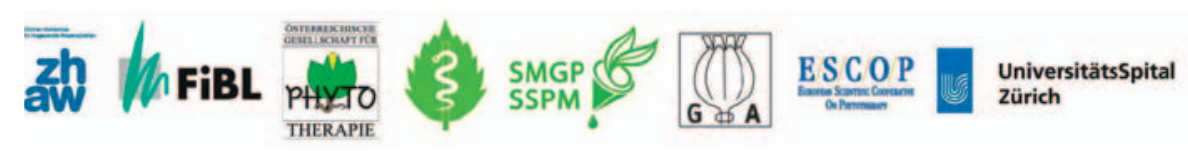

Abb. 1. Die Initiatoren der Tagung: fünf nationale und internationale Fachgesellschaften für Phytotherapie und Arzneipflanzenforschung sowie drei Institute.

Daten zur Wirksamkeit bei geriatrischen Patienten vorliegen. Immerhin zeigte die speziell für diesen Anlass erstellte Subgruppenauswertung vorliegender Studien von pflanzlichen Arzneimitteln unter Einbezug der Originaldaten, dass für Lavendelöl bei generalisierten Angsterkrankungen, subsyndromalen Angststörungen oder angstbezogenen Unruhezuständen und Schlafstörungen kein Unterschied zwischen Patienten über 60 Jahren und dem Gesamtkollektiv bestand. Dieselbe Aussage konnte für Johanniskraut (Extrakt WS 5570) anhand des HAMD(Hamilton rating scale for depression)-Gesamtscores gemacht werden. Für eine Tagesdosis von $240 \mathrm{mg}$ des Ginkgo-Extrakts EGb 761 präsentierte der Referent eine Metaanalyse von vier Studien, wobei die Zielpopulation von Ginkgo-Präparaten naturgemäss überwiegend aus älteren Patienten besteht. Sowohl in der Behandlung im kognitiven Bereich als auch in der Reduktion der Demenz-assoziierten Störungen war der Extrakt wirk-

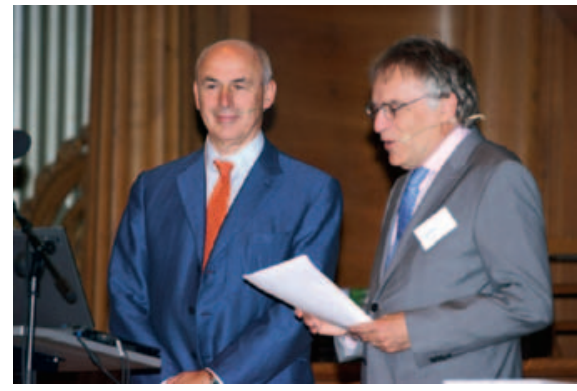

Abb. 2. Prof. Dr. Beat Meier (rechts) übernahm die einleitenden Worte zum Vortrag von Prof. Dr. med. Siegfried Kasper (links).

sam. Ebenso wurde die Belastung der Betreuungspersonen deutlich verringert.

«Mit pflanzlichen Arzneimitteln erzeugte Signale im EEG und deren Bedeutung für die Klinik in der Geriatrie»

Der Frequenzgehalt eines Elektroenzephalogramms (EEG) weist Unterschiede zwischen jüngeren und älteren Menschen auf. Ältere Personen zeigen eine signifikante Zunahme der

\section{KARGER}

Fax +4976145207 14

Information@Karger.com

www.karger.com
(๑) 2014 S. Karger GmbH, Freiburg

Accessible online at: www.karger.com/szg
Prof. Dr. sc. nat. Beat Meie

Geschäftsstelle SMGP

c/o Zürcher Hochschule für angewandte Wissenschaften

Fachgruppe Phytopharmazie, Life Sciences und Facility Management

Grüental, Postfach 335, 8820 Wädenswil, Schweiz

Beat.Meier@zhaw.ch 


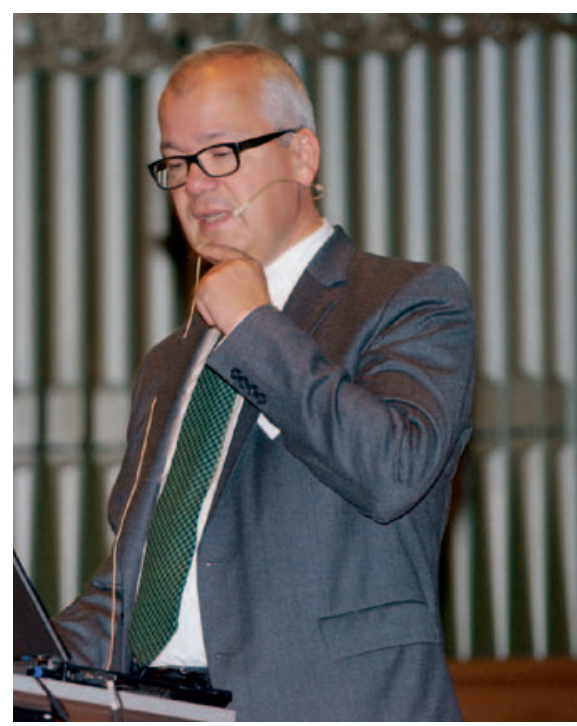

Abb. 3. Prof. Dr. med. Egemen Savaskan äusserte sich zum Thema «Ginkgo und kognitive Störungen».

schnellen Betawellen. Entsprechend reagieren sie anders auf Medikamente, die die glutamaterge und die GABAerge Übertragung angreifen. Die typischen Störungen des höheren Alters sind ein Nachlassen der kognitiven Leistungen sowie Schlafstörungen. Beide Störungen lassen sich mit einer entsprechenden Auswertung des EEGs quantifizieren, was eine gezielte Behandlung ermöglicht. Prof. Dr. med. Wilfried Dimpfel, Wetzlar, hat eine innovative Form der Schlafdarstellung entwickelt, die das Verhältnis der Theta-, Beta- und Beta2-Wellen zueinander räumlich im Ablauf der Nacht abbildet. Der Einfluss pflanzlicher Sedativa auf die Schlafqualität lässt sich damit gut darstellen. Das Elektropharmakogramm sieht der Referent als «probates Mittel» zur Objektivierung der Wirkung von pflanzlichen Präparaten. Diese ist für verschiedene Produkte erfolgt.

\section{"Ginkgo und kognitive}

Störungen: Potenzial und

\section{Praxiserfahrungen»}

Die Komplexität der Pathologie der Demenzerkrankungen macht die Entwicklung von Therapien zu einer Herausforderung. Neben den im Fokus der Forschung stehenden klassi- schen degenerativen Veränderungen (Amyloid- $\beta$-Peptid-Plaques, Veränderungen des Zytoskeletts) bieten andere pathologische Mechanismen der Neurodegeneration Ansätze für eine Therapie. Dazu gehören der oxidative Stress und Störungen in den Mitochondrien. Von den pflanzlichen Substanzen, die stark antioxidativ wirksam sind, ist der Ginkgo-bilobaExtrakt EGb 761 sehr intensiv in präklinischen und klinischen Studien untersucht worden. Unter dieser Therapie wird die Mitochondrien-Atmung verbessert, der oxidative Stress reduziert und die neurotoxische Wirkung des Amyloid- $\beta$-Peptids vermindert. Das erläuterte Prof. Dr. med. Egemen Savaskan (Abb. 3), Universitätsspital Zürich, der darauf hinwies, dass der Extrakt bisher der einzige pflanzliche Wirkstoff ist, der als Antidementivum in die Therapieempfehlungen für Demenzerkrankungen aufgenommen wurde. In den Leitlinien der Weltföderation der Gesellschaften der Biologischen Psychiatrie wird er für die Therapie der Alzheimer-Demenz und der vaskulären Demenz empfohlen.

\section{«Heilkräuter in der}

\section{Gartentherapie - Erfahrungen}

\section{aus Alterseinrichtungen»}

Die Bedeutung der Heilkräuter in der Gartentherapie präsentierte lic. phil. Thomas Pfister, Zürich. «Gartentherapie ist ein von einer ausgebildeten Gartentherapeutin oder einem Gartentherapeuten gesteuerter Prozess, in welchem Pflanzen und Gartenaktivitäten gezielt dazu verwendet werden, das soziale, psychische und körperliche Wohlbefinden von Menschen zu stärken», so lautet die Definition der Gartentherapie. Sowohl physische als auch psychische Traumata lassen sich unter Einbezug der Gartenarbeit wirksamer behandeln. Man kennt drei Ansätze der Gartentherapie: den berufsbezogenen Einsatz in der Arbeitsagogik (Wiedereingliederung und Belastbarkeitserprobung), den therapeutischen Einsatz

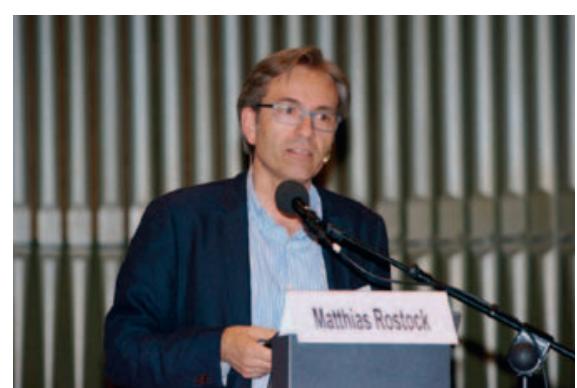

Abb. 4. Das Potenzial der Phytotherapie bei Erkrankungen der Prostata wurde von Dr. med. Matthias Rostock vorgestellt.

bei der Rehabilitation (durch Förderung der Feinmotorik z.B. von hirnverletzten Personen) und den sozialen Einsatz zur Förderung der Lebensqualität (Gesundheitsförderung). Die Gartentherapie kann vielfältig eingesetzt werden, z.B. in Alters- und Pflegezentren, in der Psychiatrie, in Institutionen für Behinderte, in Sonderschulen, in Spitälern und in Rehakliniken. In der Gartentherapie sind Heil- und Küchenkräuter vielfältig nutzbar. Zum einen vermitteln sie ein sensorisches Erlebnis, zum anderen können sie von den betreuten Personen selber in der Küche und als Heilmittel verwendet werden.

\section{«Das Potenzial der}

Phytotherapie bei Erkrankungen der Prostata mit Schwerpunkt

\section{Prostatakarzinom»}

Das Potenzial der Phytotherapie bei Erkrankungen der Prostata beleuchtete Dr. med. Matthias Rostock (Abb. 4), Hamburg und Zürich. Viele betroffene Patienten bevorzugen pflanzliche Arzneimittel, weil die synthetischen Präparate unangenehme Nebenwirkungen verursachen: Am häufigsten werden beim benignen Prostatasyndrom Extrakte aus Sägepalmenfrüchten (Serenoa repens oder Sabal serrulata), Brennnesselwurzeln (Urtica dioica), der Rinde des afrikanischen Pflaumenbaums (Pygeum africanum oder Prunus africana), Samen des Kürbisses (Cucurbita pepo) sowie Roggenpollen (Secale cereale) 
Tab. 1. Arzneipflanzen, die in der Meran-Studie in speziellen Zubereitungen eingesetzt wurden

\begin{tabular}{ll}
\hline Fatigue & Mistel, Ginseng, Eleutherococcus, Rosenwurz \\
Durchfall & Flohsamenschalen, Kombinationspräparat aus \\
& Myrrhe, Kamille und Kaffeekohle \\
Hand-Fuss-Syndrom & Leinsamenbad \\
Nervenschädigungen & Capsaicinsalbe, Leinöl \\
Schlafstörungen & Lavendelöl, Passionsblume, Baldrian \\
Wechseljahresbeschwerden & Salbei und Lavendel, Traubensilberkerze \\
Ödeme & Boswellia serrata \\
Schleimhautirritationen & Ananaswürfel, Spülungen mit Kamille, \\
& Ringelblume, Salbei, Teebaumöl, Honig, \\
& Fruchtfleischöl aus Sanddorn \\
Verstopfung & getrocknete Früchte, Aloe-Saft, \\
& Leinsamenschalen \\
Übelkeit und Erbrechen & Ingwerwurzel, Pfefferminzöl, Rosenöl, \\
& Bitterstoffe enthaltende Pflanzen in \\
& Kombination \\
\hline
\end{tabular}

und Phytosterol-Extrakte in Form von Mono- oder Kombinationspräparaten angewendet. Bis heute besteht keine etablierte Strategie zur Prävention des invasiven Prostatakarzinoms, das sich aus der hochgradig intraepithelialen Prostatahyperplasie entwickeln kann. Die protektive Wirkung von z.B. Grüntee-Extrakten ist noch $\mathrm{zu}$ wenig umfassend dokumentiert, um schlüssig empfohlen werden $\mathrm{zu}$ können. Der Entscheid zur Anwendung liegt beim Therapeuten, der dem Patientenwunsch infolge des geringen Risikos durchaus folgen kann.

\section{«Komplementärmedizin bei \\ Brustkrebs - Evaluation des \\ Pilotprojekts am Krankenhaus \\ Meran»}

Prof. Dr. med. Claudia M. Witt, Zürich, und Dr. med. Christian Thuile, Meran, stellten ein Projekt zur Evaluation komplementärmedizinischer Interventionen bei Patientinnen mit Mammakarzinom vor. Die Patientinnen wurden an einem der drei Zentren in Bozen, Brixen oder Meran behandelt. Sie wurden in eine Gruppe mit zusätzlicher komplementärmedizinischer Behandlung (CM) und eine Gruppe ohne diese Behandlung (usual care group) eingeteilt. Die zusätzliche Behandlung mit CM hat die Lebensqualität der Krebspatientinnen
Epidermis, als Rückgang der kollagenen und elastischen Fasern der Dermis und als Abnahme des Fettgewebes in der Subkutis. Die pharmakologischen Prozesse an der Haut sind grundsätzlich unabhängig von der Art des Heilmittels (Synthetikum oder Phytotherapeutikum). Wichtig ist die Penetrationsfähigkeit des Vehikels. Zur Stärkung der Altershaut sind verschiedenste pflanzliche Zubereitungen geeignet, von denen man hofft, dass sie synergistische Wirkungen bei geringerer Nebenwirkungsrate entfalten. Zur Pflege sind vor allem pflanzliche Öle (Nachtkerzenöl, Mandelöl) geeignet.

mehr verbessert als die Behandlung ohne diesen Zusatz. Die Zusatzbehandlung, bei der die Phytotherapie eine relevante Rolle spielt, wurde vom Leiter des Pilotprojekts, Christian Thuile, entwickelt. Phytotherapie wurde als Begleittherapie mit dem Ziel, die Nebenwirkungen der konventionellen Therapien zu minimieren, angeboten (Tab. 1). Festzuhalten ist, dass mit der Studie ausschliesslich der generelle Nutzen komplementärmedizinischer Massnahmen für Patientinnen mit Mammakarzinom untersucht wurde, nicht aber, ob einzelne phytotherapeutische Zubereitungen wirksam sind.

\section{«Betrachtungen zur Altershaut} mit Fokus auf die Phytotherapie» Dr. med. Oliver Das (Abb. 5), Zürich, stellte Betrachtungen zur Altershaut mit Fokus auf die Phytotherapie an. Die Altershaut ist verschiedenen morphologischen Änderungen unterworfen, die sie für bestimmte Hauterkrankungen anfällig machen. Die altersbedingten Veränderungen der Haut haben extrinsische, d.h. umweltbedingte, und intrinsische Ursachen. Die Haut wird lederartig und weist tiefe Falten, eine Gelbfärbung und aktinische Präkanzerosen auf. Histologisch erscheinen die Veränderungen als Atrophie der
«Arzneimittelinteraktionen bei multimorbiden Patienten welche Rolle spielen pflanzliche Arzneimittel?»

PD Dr. Matthias Unger, Würzburg, berichtete von einer ständig wachsenden Zahl von Publikationen zum Thema Interaktionen von pflanzlichen Arzneimitteln. Allein im Jahr 2013 fand er bei einer Literatursuche in PubMed 1681 Publikationen und 500 Übersichtsartikel! Seine Konklusionen standen dazu in einem umgekehrt proportionalen Verhältnis: Ausser bei Johanniskraut unter speziellen Bedin-

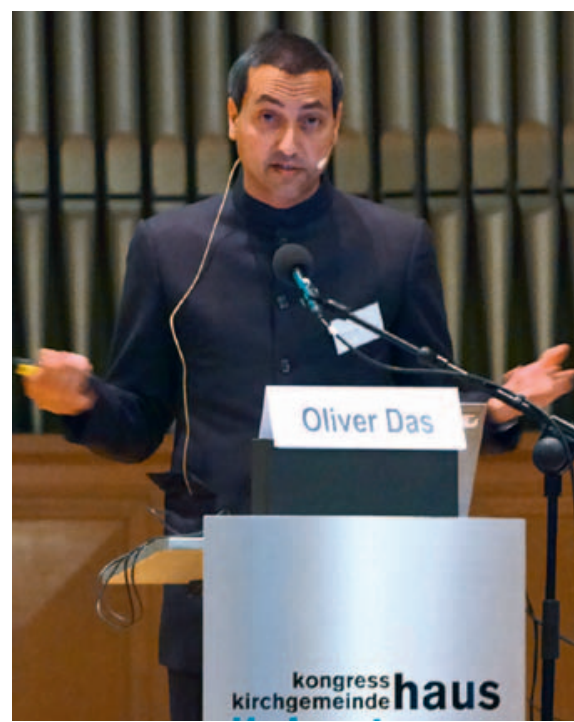

Abb. 5. Dr. med. Oliver Das stellte Überlegungen zur Altershaut mit Fokus auf die Phytotherapie an. 


\section{Fort- und Weiterbildung in Phytotherapie}

Hauptaufgabe der SMGP ist die Gewährleistung und Sicherstellung einer wissenschaftlich orientierten Ausbildung. Haben Sie Lust, an einem spannenden und abwechslungsreichen Angebot während 3 Jahren mitzumachen? Mit Ihnen noch unbekannten Kolleginnen und Kollegen in dieser Zeit Fäden zu spinnen? Die Phytotherapie als Mittler zwischen Schulund Komplementärmedizin bietet sich geradezu an, neue Wege zu beschreiten. Wir garantieren für Kurse auf hohem Niveau, spannende Diskussionen und Auseinandersetzungen. Der Bezug zur Natur geht dabei nicht vergessen: Die Pflanzen werden auf Exkursionen an ihrem natürlichen Standort betrachtet. Die Ausbildung endet mit dem Fähigkeitsausweis Phytotherapie (SMGP), der sowohl von der FMH als auch von der FPH anerkannt ist. Details zur Weiterbildung mit den Terminen und die entsprechenden Reglemente finden Sie jederzeit aktuell auf www.smgp.ch. Der Zyklus ist modular aufgebaut und ein Einstieg deshalb jederzeit möglich. Ideal für den Start ist der Grundkurs, der jeweils von Donnerstag bis Samstag in Engelberg stattfindet. 2015 wird dieser Kurs vom 28. bis 30. Mai angeboten.

gungen fand er keine relevanten Interaktionen von arzneibuchkonform hergestellten Extrakten und Zubereitungen mit einer klinischen Relevanz! Aus den vielen experimentellen Daten können keine In-vivo-Daten abgeleitet werden, da die In-vitro/In-vivo-

\section{Bilanz}

Die Phytotherapie hat in der Altersmedizin ein erhebliches Potenzial. Diese Bilanz kann nach dieser Tagung gezogen werden. Mit vereinten Kräften sollte diese Position gefestigt und gestärkt werden. Deshalb ermöglicht das Supplementheft 1/2014 der Zeitschrift Forschende KOMPLEMENTÄRMEDIZIN eine vertiefte Beschäftigung mit dem Thema «Phytotherapie in der Geriatrie und Gerontologie» auch nach der Tagung. Die Texte der Referierenden sind online frei zugänglich unter: www.karger.com/Journal/ Issue/261752. Der hier vorliegende Bericht wurde auf der Basis des gesprochenen Wortes und mithilfe der schriftlichen Fassungen der Referierenden in dieser Ausgabe zusammengestellt. Weitere Informationen finden sich auf der Website der Tagung (http://phytotherapie2014.smgp.ch). Hier können unter anderem auch viele der präsentierten Poster eingesehen werden.
Korrelation fehlt. Daraus kann gefolgert werden, dass die Substitution synthetischer Arzneimittel durch pflanzliche Zubereitungen nur ein minimales Interaktionspotenzial aufweist. 